\title{
Photosensitive heterostructures CdTe-PbTe prepared by hot-wall technique
}

\author{
S. Movchan, F. Sizov, V. Tetyorkin \\ Institute of Semiconductor Physics of NASU, 45, prospect Nauki , 252028 Kiev, Ukraine
}

\begin{abstract}
Hot-wall technique has been used for preparation of $\mathrm{CdTe}-\mathrm{PbTe}$ heterostructures. $\mathrm{BaF}_{2}$ single crystals served as substrates. Electrical, photoelectric properties as well as noise spectra were investigated. Heterostructures exhibit photosensitivity up to room temperatures in the middle infrared (IR) region. In the heterostructures investigated at room temperature the $1 / f$ noise is observed at frequencies much less compared to those ones observed in PbSe photoresistors $(f \approx 3000 \mathrm{~Hz})$ for the same IR region. Carrier transport mechanisms and band diagram of the heterostructures are briefly discussed.
\end{abstract}

Keywords: narrow-gap semiconductors, heterostructures, photoresponse spectra, carrier transport mechanisms.

Paper 02.07.99; revised manuscript received 05.07.99; accepted for publication 12.07.99.

\section{Introduction}

$\mathrm{A}_{2} \mathrm{~B}_{6}$ and $\mathrm{A}_{4} \mathrm{~B}_{6}$ semiconductors are recognised to be the most important materials for manufacturing modern infrared (IR) devices. Among these CdTe and PbTe are well investigated ones. Both CdTe and PbTe epitaxial layers can be grown by hot wall epitaxy (HWE) technique [1,2]. An advantage of the HWE technique consists in a possibility of preparing epitaxial layers of high quality at rather low growth temperature (less than $T \approx 400$ ) during a relatively short period of a time. As a rule, the epitaxial layers do not require thermal annealing to decrease density of native defects. This allows one to prepare heterostructures on their base as well as multiple quantum wells and superlattices [3]. Applied interest to the heterostructures pointed out stems from their potential usage as IR photon detectors for the middle $(3 \div 5 \mathrm{~mm})$ IR region. They can also attract great scientific interest. The reason is, these semiconductors have different crysral lattice structures [3] and their physical properties (band gaps, static dielectric constants, lattice constants, effective masses of carriers, etc.) differ much (see Table 1). It means that the variety of the physical phenomena are to be observed in these structures.

Despite the fact that the circumstances listed above seem to be not appropriate for the photosensitive heterostructures preparation, successful attempt to manufacture these structures was demonstrated here.

It should be pointed out that the data published so far are rather scarse. In [4] PbTe-CdTe-InSb heterostructures and $\mathrm{CdTe}-\mathrm{PbTe} \mathrm{MQWs}$ were prepared by ionized-cluster beam epitaxy. Heterostructures $\mathrm{Pb}_{1-x} \mathrm{Sn}_{x} \mathrm{Te} / \mathrm{CdTe}$ were also investigated [5].

Table 1. Physical properties of CdTe and PbTe single crystals

\begin{tabular}{lcc}
\hline \hline & $\mathrm{CdTe}$ & $\mathrm{PbTe}$ \\
\hline Lattice structure & cub. $(\mathrm{ZnS})$ & cub. $(\mathrm{NaCl})$ \\
Lattice constant & & \\
$a, 300 \mathrm{~K}, \mathrm{~nm}$ & 0.6478 & 0.6460 \\
Energy gap & & \\
$E_{g}, \mathrm{eV}, 300 \mathrm{~K}$ & 1.50 & 0.32 \\
\multicolumn{1}{c}{$77 \mathrm{~K}$} & 1.60 & 0.22 \\
$\partial E_{g} / \partial T, 10^{-4} \mathrm{eV} / \mathrm{K}$ & -4.1 & 4.2 \\
Electron affinity & & \\
$\chi, \mathrm{eV}, 300 \mathrm{~K}$ & 4.3 & 4.6 \\
\hline \hline
\end{tabular}

The purpose of the present paper is to prepare CdTePbTe heterostructures by HWE technique and to study their basic characteristics.

\section{Heterostructure technology}

Epitaxial layers were deposited in vacuum system $\left(10^{-5} \mathrm{~Pa}\right)$ similar to that one, described in detail elsewhere [6]. The system is provided by two independent sources. The growth 


\section{S. Movchan et al.: Photosensitive heterostructures CdTe-PbTe...}

conditions are the following: PbTe and CdTe source temperature $-440 \div 470{ }^{\circ} \mathrm{C}$, and substrate temperature $-380 \div 400{ }^{\circ} \mathrm{C}$. $\mathrm{BaF}_{2}$ single crystals cleaved along (111) direction immediately before the deposition serve as substrates. Optical and electrical properties of epitaxial layers were examined on separate samples grown on $\mathrm{BaF}_{2}$ substrates under the same growth condition.

In PbTe layers grown from single crystals electron and hole concentration were determined to be in the range of $(1 \div 6) 10^{17} \mathrm{~cm}^{-3}$, and their mobilities were ranged from 2300 to $10000 \mathrm{~cm}^{2} / \mathrm{V} \times \mathrm{s}$ at $77 \mathrm{~K}$. Doped with $\mathrm{Cl}$ and undoped single crystals of CdTe has been used for deposition. Specific resistance of CdTe layers was estimated to be $10^{4}$ $10^{5} \mathrm{Ohm} \times \mathrm{cm}$ and electron concentration was about $10^{13} \mathrm{~cm}^{-3}$ as in the single crystals used for the sources. The thickness of the layers was in the range of $(2.5 \div 4.0) \mathrm{mm}$.

The mesa structures have been prepared by using evaporation through the stainless steel masks. The mesa structures had an area $1.7 \times 10^{-3} \mathrm{~cm}^{2}$. Ohmic contacts to n-type $\mathrm{PbTe}$ and CdTe layers were prepared by thermal deposition of indium.

\section{Experimental results and discussion}

Typical current-voltage characteristics of $(n) \mathrm{PbTe}-(n) \mathrm{CdTe}$ heterostructures measured at room temperature as well as at $T=77 \mathrm{~K}$ are shown in Fig. 1 . As it is seen they have asymmetric character at both temperatures. The current saturation at reverse bias voltage are not observed.

Typical capacitance-voltage characteristic is illustrated in Fig.2. One should point out weak dependence of the capacitance on the voltage bias. Previously similar behavior of the high-frequency capacitance was observed in $(n) \mathrm{Si}-(n) \mathrm{Ge}$ heterostructures [7]. The dependence $C v s U$, such as shown in Fig.2, seems to be typical for isotype heterojunctions with two depleted regions. The capacitance values are reasonably small for such large area heterostructures which implies their use at relatively high frequency compared with $\mathrm{PbSe}$ and $\mathrm{PbS}$ pfotoresistors which can be effectively used only in the range of $f \leq 5 \cdot 10^{4} \mathrm{~Hz}$.

Typical photoresponse spectra of the heterostructure measured at zero bias are shown in Fig.3 at room temperature and $T=82 \mathrm{~K}$. It should be pointed out that they differ significantly from the characteristics observed in lattice matched heterostructures [7]. The following peculiarities of the spectra are to be noticed. The spectrum at each temperature consists of two parts. The sign of the detected signal was the same for both parts of the spectra measured at room temperature as well as at the temperature $T=82 \mathrm{~K}$. The long-wavelength edge of the photoresponse poorly depends on temperature.

Because of PbTe and CdTe have different type of lattice structures (see Table 1), one can assume significant number of the interface states in the heterostructures on their basis. These states can influence carrier transport mechanisms, capacitance-voltage characteristics, photoelectrical properties as well as band diagram of these structures.

The total dark current of the heterostructures can be
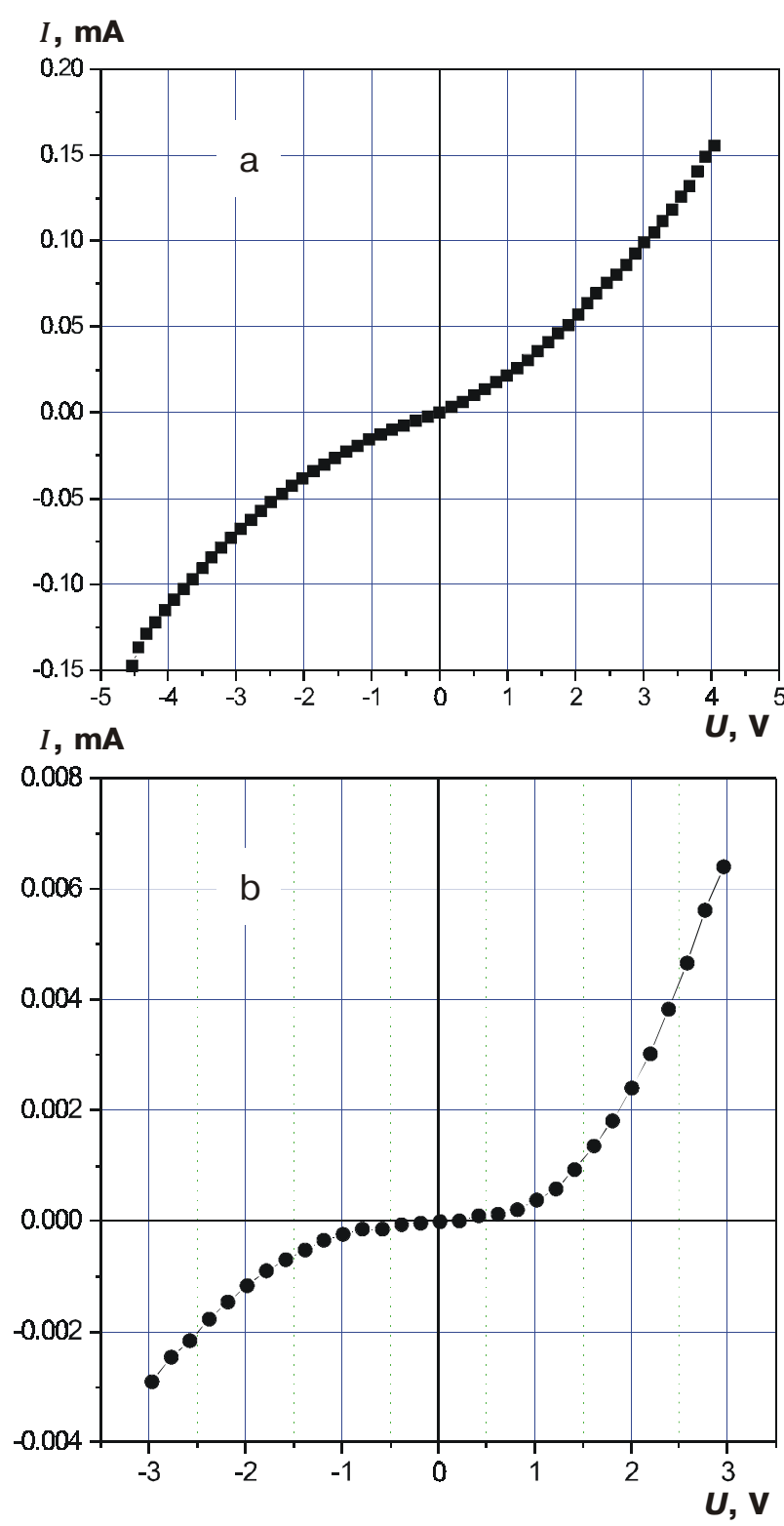

Fig.1. Current-voltage characteristics of $(n) \mathrm{CdTe}-(n) \mathrm{PbTe}$ heterostructure at temperatures $77 \mathrm{~K}$ (a) and $300 \mathrm{~K}$ (b). Positive potential is applied to PbTe.

represented as a sum of several components

$J=J_{G R}+J_{d}+J_{T}+J_{L}$,

where the symbols denote accordingly: generationrecombination current, diffusion current, tunneling current, surface leakage current, and emission current. If generation-recombination and diffusion mechanisms are dominant the dark current obeys the law $J=J_{0} \exp [(q U / \beta k T)-1]$, where the $\beta$ coefficient is changed from 1.0 to 2.0. Such values were observed in lattice matched heterostructures based on PbTe and PbSnTe compounds [3]. However, it has been established that in the heterostructures investigated the forward current can be expressed as $J=J_{\mathrm{o}}$ [exp $\left.(A U)-1\right]$ for bias voltages $U \leq 2.0 \mathrm{~V}$, where the coefficient $A$ is ranged 


\section{S. Movchan et al.: Photosensitive heterostructures CdTe-PbTe...}

from 2 to 5. It means that tunneling is the dominant carrier transport mechanism [7]. For $U>2.0 \mathrm{~V}$ the current-voltage characteristics are linear due to influence of large series resistance, which consist of the resistance of neutral region of the CdTe layer, resistance of the contacts, etc. From the intercept of the linear part of $J$ vs $U$ characteristics with the voltage axis the values of the diffusion potential $U_{D J}=1.2$ - 1.4 were determined. The lack of the current saturation at reverse bias can also be explained by tunneling mechanism. Further, in heterostructures investigated the tunnel current can consist of two components: band-to-band tunnel current and tunnel current through deep defect states in the gap. The preliminary analysis testifies in favour of the last mechanism.

As a rule, interface states result in formation of double depletion regions and in accordance with the Anderson model heterostructure can be represented as two Schottky diodes switched towards each other [7]. Equivalent electric circuit of the heterojunction is shown in Fig.2. The five components in this circuit are voltage- and frequency-dependent and capacitance can be rather complicated function of bias voltage and frequency. An analysis of this circuit for zero series resistance is given in [8]. Because of $R_{s}$ together with differential resistance of each diodes governs the distribution of applied voltage it can influence both current-voltage and capacitance-voltage characteristics. This seems to be the reason why the dependence of the capacitance on the bias voltage is rather weak in the heterostructures investigated, Fig.2. One can estimate highfrequency capacitance from the formula [8]

$$
C=\frac{C_{C d T e} \cdot C_{P b T e}}{C_{C d T e}+C_{P b T e}},
$$

were $C_{C d T e}$ and $C_{P b T e}$ are the capacitances of appropriate diodes. It is clear that $C_{P b T e}>>C_{C d T e}$ and $C \cong C_{C d T e}$. At zero bias $C_{C d T e}$ can be calculated from the relation [7]

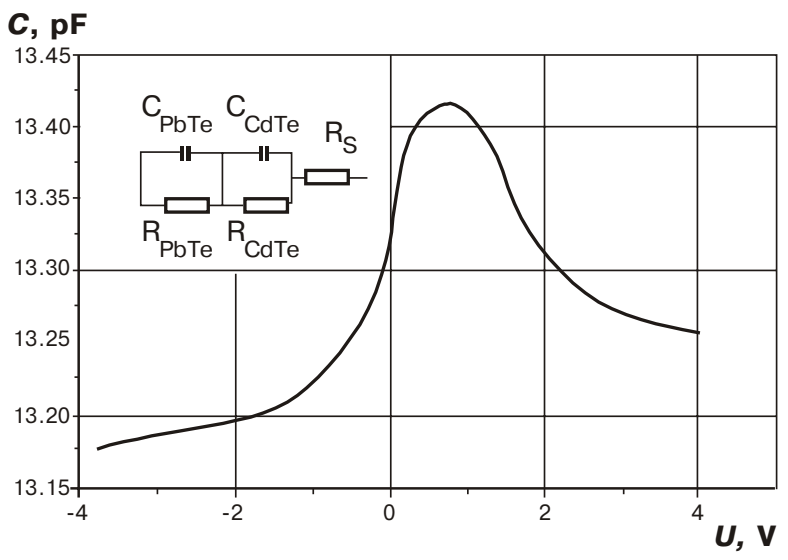

Fig.2. Capacitance-voltage characteristics $C-U$ of the heterostructure at a temperature $77 \mathrm{~K}$ at frequency $1 \mathrm{MHz}$. Insert shows the equivalent circuit for the model of the heterojunction.
$C_{C d T e}=\left[\frac{q \varepsilon_{0} \varepsilon n}{2 U_{D}}\right]^{1 / 2} \cdot A$.

It was established that the values of the capacitance observed can be explained for electron concentration $n \leq 10^{14} \mathrm{~cm}^{-3}$ in accordance with the data measured in separate CdTe layers.

In the model of two Schottky diodes photoresponse spectrum also consists of two parts due to generation of electron-hole pairs in narrow- and wide-gap semiconductors. Obviously, the signal detected by set-up from these diodes should have an opposite sign. It is well-known fact for (n) Ge- $(n) \mathrm{Si}$ heterostructures [7]. However, in the heterostructures under investigation it has not been observed. One can assume that long-wavelength part of the photoresponse spectra shown in Fig. 3 is caused by excitation of carriers from the interface states. The excitation energy $h \omega_{e x}$ has to be not less than $\Delta E_{c}$, where $\Delta E_{c}$ is the conduction band discontinuity. In the first approximation $\Delta E_{c}$ is equal to electron affinity difference $\Delta \chi=\chi_{P b T e}-\chi_{C d T e}$ $=0.3 \mathrm{eV}$ (see Table 1). This value coincides well with the energy of the long-wavelength photoresponse edge 0.26 $0.28 \mathrm{eV}$, Fig.3. Furthermore, its weak dependence on temperature can be explained in the framework of the approximation used if one take into account that electron affinity may have weak dependence on temperature.
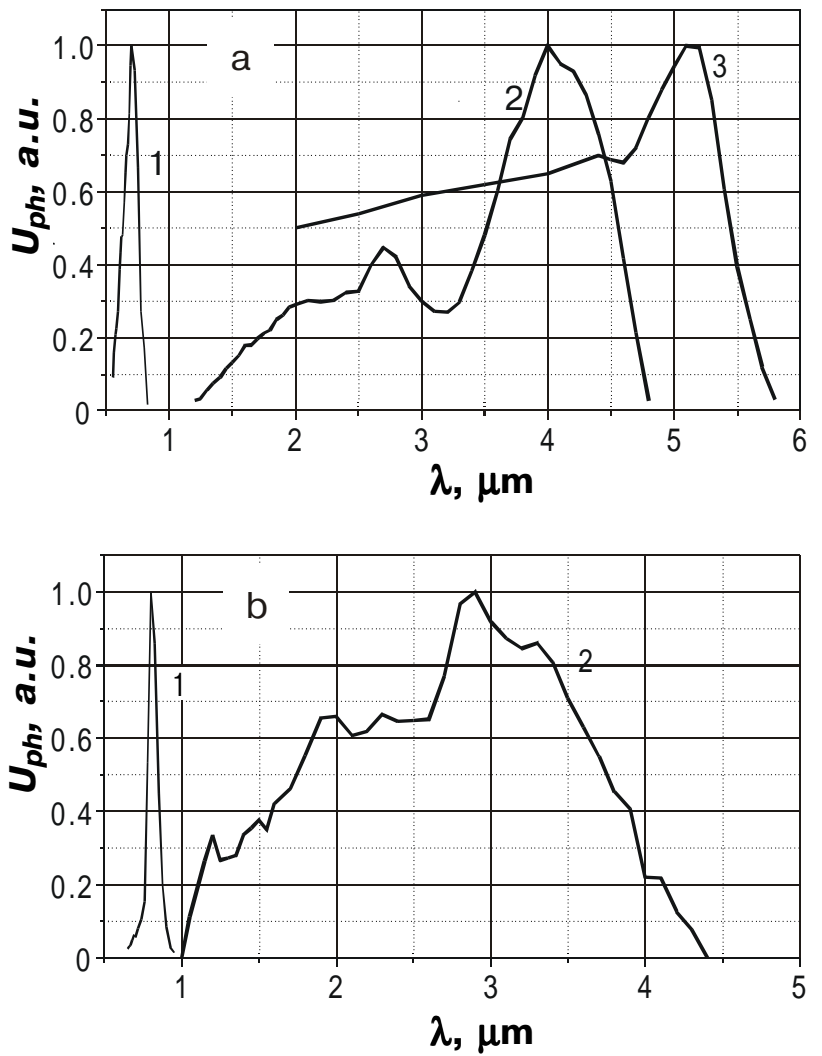

Fig.3. Photoresponse spectra at temperatures $82 \mathrm{~K}$ (a) and $300 \mathrm{~K}$ (b). Both parts of the spectra (curves 1 and 2) are normalized separately. Also shown is the photoresponse spectrum of the $\mathrm{Cu}-\mathrm{PbTe}$ Schottky diodes at $82 \mathrm{~K}$ (a, curve 3 ). 


\section{S. Movchan et al.: Photosensitive heterostructures CdTe-PbTe...}

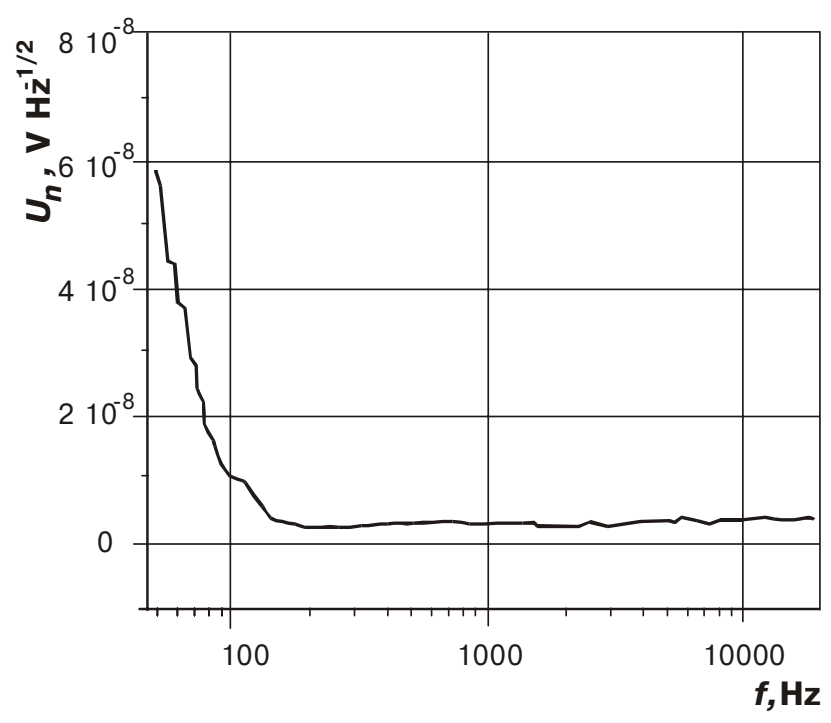

Fig.4. Typical noise spectrum of the heterostructure at a room temperature.

Another explanation of the data analysed above consist in existence of intermediate layer at the interface due to interdiffusion of cadmium and lead atoms. One can suppose that interdiffusion results in formation of $\mathrm{PbCdTe}$ solid solution. Obviously, its energy gap should be higher as well as the value of $\partial E_{g} / \partial T$ should be lower than in the lead telluride (see Table 1). The data shown in Fig. 3 a supports this supposition. So, to clarify experimental results additional investigations are needed.

Shown in Fig.4 is the typical noise spectrum measured at room temperature. As it is seen, the $1 / f$ noise at $T=300 \mathrm{~K}$ is observed at frequencies less than $200 \mathrm{~Hz}$ and is much less the typical frequency for $\mathrm{PbSe}$ photoresistors $(f \approx 3000 \mathrm{~Hz}$ ) [9]. This favors possible application of the heterostructures investigated for the same spectral range in the infrared optoelectronics. At $T=80 \mathrm{~K}$ the $1 / f$ noise begins at $f \approx 80 \mathrm{~Hz}$ that is at a substantially lower value compared to $\mathrm{PbSe}$ photoconductors $(f \approx 300 \mathrm{~Hz}[9])$.

\section{Conclusion.}

It has been demonstrated that sensitive CdTe-PbTe heterostructures can be grown by hot-wall epitaxy method. Current-voltage and capacitance-voltage characteristics as well as photoresponse and noise spectra were investigated in the heterostructures for the first time. The heterostructures have been shown to be photosensitive up to room temperature and demonstrated the level of $1 / f$ noise at much lower frequencies compared to $\mathrm{PbSe}$ photoresistors for the same middle wavelength spectral band.

\section{References}

1. A.A. Tikhonova, Ye.B. Orlova, U. Suekhua, D.Ye. Sklovski. Peculiarities of $\mathrm{CdTe} /(001) \mathrm{GaAs}$ epitaxy under deposition using «hot-wall» technique. Izvestiya Akademii Nauk, ser. fizicheskaya, 1993, v.57, N 2, p.120-125 (in Russian)

2. I. Sugijama, A. Hobbs, K. Shinohara, and H. Takigawa. Structural properties of CdTe-ZnTe strained-layer superlattice grown on GaAs by hot-wall epitaxy. Appl. Phys. Lett., 1991, v.58, N24, P.2755-2757.

3. Infrared Photon Detectors. Ed. by A. Rogalski. SPIE Optical Engineering Press, 1995.

4. Toshinori Takagi, Hiroshi Takaoka, and Youichi Kuriyama. Ionizedcluster beam epitaxy of CdTe and its application to $\mathrm{CdTe} / \mathrm{PbTe}$ multilayer structure. Thin Solid Films, 1985, v.126, P.149-154.

5. S.D. Darchuk, S.V. Plyatsko, F.F. Sizov, M.V. Apatskaya, O.A. Yakubtsov. Processes of a current transport in heterojunctions basen on narrow-gap semiconductor $\mathrm{Pb}_{1-x} \mathrm{Sn}_{x} \mathrm{Te} / \mathrm{CdTe}$. Optoelectronica $i$ poluprovonikovaya tekhnika, 1989, 16, N 84-87 (in Russian)

6. A. Lopez-Otero. Hot wall epitaxy. Thin Solid Films. 1978, V.491, P.354

7. B.L. Sharma and R.K. Purohit. Semiconductor heterojunctions.Pergamon Press, 1974.

8. C. Van Opdorp and H.K.J.Kanerva. Current-voltage characteristics and capacitance of isotype heterojunctions. Solid-State Electronics, 1967, v.10, N5, p.401-421.

9. E.L. Dereniak and G.D. Boreman. Infrared Detectors and Systems. J. Wiley and Sons. N-Y, 1996. 